\title{
mTORC1 stimulates nucleotide synthesis through both transcriptional and post-translational mechanisms
}

\author{
Issam Ben-Sahra ${ }^{1 *}$, Stephane Ricoult ${ }^{1}$, Jessica Howell ${ }^{1}$, John Asara ${ }^{2}$, Brendan Manning ${ }^{1}$ \\ From Metabolism, Diet and Disease 2014: Cancer and metabolism \\ Washington DC, USA. 28-30 May 2014
}

\section{Background}

Cellular growth signals stimulate anabolic processes. The mechanistic target of rapamycin (mTOR), as part of mTORC1, is a protein kinase that senses growth signals to regulate anabolic growth and proliferation. mTORC1 stimulates protein synthesis through effects on mRNA translation and ribosome biogenesis [1]. mTORC1 signaling also promotes de novo lipid and sterol synthesis through the activation of the sterol-response element-binding protein (SREBP) transcription factors, which stimulate the expression of the enzymes driving this biosynthetic process [2].

\section{Material and Methods}

TSC2 ${ }^{+/+}$MEFs, TSC2 $2^{-/-}$MEFs, MCF10A expressing pBabe-empty vector or PI3KCA ${ }^{\mathrm{H} 1047 \mathrm{R}}$, HeLa cells, U87MG cell line were used in this study. To determine the relative levels of intracellular metabolites, extracts were prepared and analyzed by LC/MS/MS [3]. Regarding the $\mathrm{U}^{14} \mathrm{C}$-aspartate, $\mathrm{U}$-14C-glycine incorporation into RNA and DNA, cells were serum starved for 15 hours and treated as indicated. Cells were harvested and RNA or DNA was isolated using Allprep DNA/RNA kits according to the manufacturer's instructions and quantified using a spectrophotometer. For statistical analysis a two-tailed Student's t-test was performed for all pairwise comparisons $(n=3)$.

\section{Results}

We find that activation of mTORC1 leads to the acute stimulation of metabolic flux through the de novo pyrimidine synthesis pathway [4]. We recently found that

${ }^{1}$ Harvard School of Public Health, Department of Genetics and Complex Diseases, Boston, MA, USA

Full list of author information is available at the end of the article
mTORC1 stimulates the de novo purine synthesis pathway. In contrast with pyrimidine synthesis, the regulation of the purine synthesis by mTORC1 signaling occurs through long-term mechanism. Indeed, we found that mTORC1 regulates the de novo purine synthesis pathway through the transcription factor SREBP.

\section{Conclusion}

These findings demonstrate that growth signaling through mTORC1 promotes the production of new nucleotides to facilitate an increased demand for RNA and DNA. mTOR appears to be a central regulator of de novo nucleotide synthesis. Therefore, nucleotide synthesis joins protein and lipid synthesis as major anabolic processes stimulated by mTORC1 signaling.

\section{Authors' details}

${ }^{1}$ Harvard School of Public Health, Department of Genetics and Complex Diseases, Boston, MA, USA. ${ }^{2}$ Division of Signal Transduction, Beth Israel Deaconess Medical Center and Department of Medicine, Harvard Medical School, Boston, MA, USA.

\section{Published: 28 May 2014}

\section{References}

1. Laplante M, Sabatini DM: mTOR signaling in growth control and disease. Cell 2012, 149:274.

2. Düvel $K$, et al: Activation of a metabolic gene regulatory network downstream of mTOR complex 1. Mol Cell 2010, 39:171.

3. Yuan $\mathrm{M}$, et al: A positive/negative ion-switching, targeted mass spectrometry-based metabolomics platform for bodily fluids, cells, and fresh and fixed tissue. Nat Protoc 2012, 7:872.

4. Ben-Sahra I, et al: Stimulation of de novo pyrimidine synthesis by growth signaling through mTOR and S6K1. Science 2013, 339:1323-1328.

\section{doi:10.1186/2049-3002-2-S1-P6}

Cite this article as: Ben-Sahra et al:: mTORC1 stimulates nucleotide synthesis through both transcriptional and post-translational mechanisms. Cancer \& Metabolism 2014 2(Suppl 1):P6. 